Research Article

\title{
Effect of Antifungal-Treated Host Macrophages on Candida glabrata
}

\author{
Hong-Bin Li, ${ }^{1}$ Na Li, ${ }^{1}$ Shu-Ran Wen, ${ }^{1}$ Ming-Yue Qiang, ${ }^{1}$ Zheng-Hui Yang, \\ Tian-Xiang Dong, ${ }^{1}$ Yu-Ye Li, ${ }^{1,2}$ and Yi-Qun Kuang $\mathbb{D D}^{2,3}$ \\ ${ }^{1}$ Department of Dermatology and Venereology, First Affiliated Hospital of Kunming Medical University, Kunming 650032, \\ Yunnan, China \\ ${ }^{2}$ NHC Key Laboratory of Drug Addiction Medicine, First Affiliated Hospital of Kunming Medical University, \\ Kunming Medical University, Kunming 650032, Yunnan, China \\ ${ }^{3}$ Scientific Research Laboratory Center, First Affiliated Hospital of Kunming Medical University, Kunming 650032, Yunnan, \\ China
}

Correspondence should be addressed to Yi-Qun Kuang; yq610433@hotmail.com

Received 27 September 2020; Revised 7 February 2021; Accepted 9 February 2021; Published 18 February 2021

Academic Editor: Louis Detolla

Copyright (c) 2021 Hong-Bin Li et al. This is an open access article distributed under the Creative Commons Attribution License, which permits unrestricted use, distribution, and reproduction in any medium, provided the original work is properly cited.

Objective. Candida glabrata (C. glabrata) causes infections associated with severe sepsis and high mortality. This study describes the effects of micafungin (MCF), itraconazole (ICZ), and amphotericin B (AmB) on the function of macrophages during C. glabrata infection. Methods. RAW264.1 macrophages were treated with MCF, ICZ, or AmB and then challenged with C. glabrata. Cytokines from infected macrophage supernatants and the levels of superoxide dismutase (SOD) in macrophages were measured at different time points after phagocytosis. Results. The activity of SOD was significantly increased in RAW264.1 cells that phagocytized C. glabrata and reached a peak level at 6 hours $(P<0.05)$. ICZ and AmB did not affect the SOD activity in cells that phagocytized C. glabrata versus that in untreated macrophage. C. glabrata stimulated macrophages to secrete cytokines. Neither ICZ nor AmB affected the secretion of interleukin-6 (IL-6), interleukin-8 (IL-8), or tumor necrosis factor- $\alpha$ (TNF- $\alpha$ ) by C. glabrata-infected macrophages. However, MCF downregulated the secretion of TNF- $\alpha$ by infected macrophages and reduced the SOD activity of C. glabrata compared with those in untreated controls. Conclusion. Echinocandins may increase their antifungal efficacy by altering the innate immune response of macrophages and attenuating antioxidants of this organism.

\section{Introduction}

Candida is generally regarded as a nonpathogenic symbiotic microbe of human mucosal tissue that occasionally causes opportunistic infections. Previous epidemiological studies from 2005 showed that the proportion of candidemia caused by Candida glabrata (C. glabrata) in public hospitals did not exceed $5-8 \%[1,2]$. However, with the increasing use of various iatrogenic catheters, immunosuppressive agents during organ transplantation and anticancer treatment, broad-spectrum antibiotics, and the increase in HIV infection, infections caused by Candida spp. have tended to be more invasive in deep organs and manifest as sepsis, fungemia, and other life-threatening conditions. Candidemia caused by C. glabrata accounts for $10.6 \%$ of fungemia [3].

Infection caused by C. glabrata has a high mortality rate of $40-70 \%$ [4]. In recent years, the success of azole antifungal agents for the control of fungal infection has been consistent with the increase in non-Candida albicans (C. albicans) infection, among which C. glabrata infection has increased by three times. According to the ARTEMIS Global Antifungal Monitoring Project, C. glabrata accounted for 10.2\%$11.7 \%$ of all Candida spp. strains isolated from June 1997 to December 2007 [5]. A multicenter prospective survey of severe invasive Candida infections in China also showed that 
the incidence rate of C. glabrata in China was $12.9 \%$ [6]. It has been shown that C. glabrata is resistant to most antifungal drugs [7]. However, the involved mechanism is not fully understood.

Macrophages, as the first line of defense against invading pathogens, produce an oxidative stress response against pathogens while upregulating the expression of various proinflammatory cytokines and chemokines such as interleukin-6 (IL-6), interleukin-8 (IL-8), and tumor necrosis factor- $\alpha$ (TNF- $\alpha$ ), thus improving the ability to kill pathogens. C. albicans survives in macrophages through the formation of hyphae and mycelia that penetrate the cell membrane [8-10], which causes significant toxicity to macrophages. C. glabrata cannot form hyphae and has no obvious toxicity to macrophages. Studies have found that C. glabrata does not cause significant cytotoxicity in macrophages within 48 hours but can survive in macrophages for a long time [11], indicating that C. glabrata can adapt to the harsh intracellular environment and evolve strategies for intracellular survival. The antioxidant system of C. glabrata can resist damage and can be used to avoid being killed by phagocytes. The antioxidant system of C. glabrata includes catalase, glutathione peroxidase, superoxide dismutase (SOD), and glutathione, and all of which are effectors of oxidative stress. SOD is very important for many pathogenic bacteria and fungi, such as C. albicans, Histoplasma capsulatum ( $H$. capsulatum), and Cryptococcus neoformans (C. neoformans) [12-14]. It also plays an important role in the metabolism of C. glabrata and is related to the integrity of DNA and the prevention of aging [15]. In this study, we aimed to examine the role of macrophages in C. glabrata infection and treatment.

\section{Materials and Methods}

2.1. C. glabrata Strains. The C. glabrata standard strain ATCC2001 was purchased from the American Type Culture Collection (Sinozhongyuan Ltd., Beijing, China). Ten clinical strains of C. glabrata were from the Laboratory Department of First Affiliated Hospital of Kunming Medical University. All C. glabrata strains were incubated in the yeast extract-peptone-dextrose (YPD) medium (1\% yeast extract (OXOID, Britain), 2\% peptone (OXOID, Britain), and 2\% dextrose (Beijing Second Chemical Reagent Factory, Beijing, China)) or Sabouraud agar (1\% peptone, $4 \%$ glucose, $1.2 \%$ agar, and $0.025 \%$ chloramphenicol) (Bio Mérieux, Lyon, France) at $35^{\circ} \mathrm{C}$.

2.2. Antifungal Agents. MCF (GlpBio, China), ICZ (Tixiai Chemical Industry Development Co., Ltd., Shanghai, China), and AmB (Tixiai Chemical Industry Development Co., Ltd., Shanghai, China) powder at laboratory levels were obtained and used for the preparation of stock and working solutions. The minimum inhibitory concentrations (MICs) of 11 strains against three drugs were determined by the M27-A2 method according to the National Committee for Standardization of Clinical Laboratories (NCCLS) [16].
2.3. Macrophages. The macrophage cell line RAW264.7 was a gift from the Department of Immunology of Kunming Medical University. The cells were cultured in a DMEM medium (Thermo Fisher, USA) with 10\% FBS (Sangon Biotech, Shanghai, China) in an incubator containing 5\% $\mathrm{CO}_{2}$ at $37^{\circ} \mathrm{C}$. For each experiment, macrophages were pretreated with each antifungal drug at less than each MIC value and the plate was then incubated for 24 hours. After this pretreatment, C. glabrata $\left(2 \times 10^{6} / \mathrm{mL}\right)$ was added into each culture flask with $1 \mathrm{~mL}$ macrophages $\left(2 \times 10^{6}\right.$ cells $\left./ \mathrm{mL}\right)$ at an MOI $=1$ [17]. The end points for the evaluation of each phagocytosis experiment are indicated in the Result section.

2.4. Determination of Cytokines and SOD Activities. To quantify cytokines, IL-6, IL-8, and TNF- $\alpha$ in supernatants from medium or yeast-stimulated RAW264.1 macrophages were harvested by centrifugation and stored at $-80^{\circ} \mathrm{C}$ until analysis.

Infected macrophages were washed with PBS (phosphate-buffered saline, $1.06 \mathrm{mM} \mathrm{KH}_{2} \mathrm{PO} 4,155.17 \mathrm{mM} \mathrm{NaCl}$, $2.97 \mathrm{mM} \mathrm{Na}_{2} \mathrm{HPO}_{4}-7 \mathrm{H}_{2} \mathrm{O}$ ) (Thermo Fisher, USA) to remove extracellular yeast; monolayer cells were examined under a microscope to ensure that they were intact and free of extracellular yeast. Cells were then treated with cold deionized water to breakdown and release C. glabrata cells that were phagocytosed. The amounts of IL-6, IL- 8 , and TNF- $\alpha$ were measured by specific commercial ELISA kits (Solarbio, Beijing, China) according to the manufacturer's instructions. The activity of SOD was determined by a SOD activity detection kit according to the manufacturer's instructions (Solarbio, Beijing, China).

2.5. Statistics. The results were obtained from at least three independent experiments and subjected to statistical analysis. Data are presented as the mean \pm SD and were analyzed by ANOVA or Student's $t$-test by using Prism 8 software (GraphPad, San Diego, CA). The level of statistical significance was set at $P<0.05$.

\section{Results}

3.1. General Information. A total of 10 clinical strains tested in this study were from adult patients recently diagnosed at our hospital. Among 10 infected patients, 6 patients were female. Of these, two women suffered from uterine fibroid, one from renal calculus and one from cervical carcinoma. Three C. glabrata strains in the male group were isolated from patients with jaundice and intestinal obstruction. The source of 10 clinical strains and detailed clinical information are shown in Table 1.

3.2. Drug Sensitivity of Clinical Strains. To determine the optimal concentration of each antifungal agent to examine macrophage activity, the susceptibility of 11 experimental strains to MCF, ICZ, and AmB was measured according the M27-A2 protocol of NCCLS and is shown in Table 2. Compared with the MICs of ATCC2001 for AmB (0.5 mg/L) 
TABLE 1: Clinical information of clinical strains.

\begin{tabular}{lccccc}
\hline No. & Sample number & Age & Sex & Diagnosis & Source of specimen \\
\hline 1 & $17 \mathrm{~K} 1156$ & 52 & $\mathrm{~F}$ & Uterine fibroid & Uterine drainage fluid \\
2 & $\mathrm{~J} 1$ & 56 & $\mathrm{M}$ & Jaundice & Bile \\
3 & $17 \mathrm{~K} 1173$ & 42 & $\mathrm{~F}$ & Renal calculus & Peritoneal draining liquid \\
4 & $17 \mathrm{~K} 1152$ & 80 & $\mathrm{M}$ & Intestinal obstruction & Peritoneal draining liquid \\
5 & $17 \mathrm{~K} 1150$ & 51 & $\mathrm{~F}$ & Uterine fibroid & Uterine drainage fluid \\
6 & $16 \mathrm{~K} 1132$ & 27 & $\mathrm{M}$ & Jaundice & Bile \\
7 & $16 \mathrm{~K} 1128$ & 38 & $\mathrm{~F}$ & Cervical carcinoma & Pus \\
8 & $17 \mathrm{~S} 709$ & 25 & $\mathrm{~F}$ & Candidal vaginitis & Leucorrhea \\
9 & $16 \mathrm{~S} 482$ & 45 & $\mathrm{~F}$ & Candidal vaginitis & Leucorrhea \\
10 & $16 \mathrm{~S} 506$ & 60 & $\mathrm{M}$ & Deep fungal infection & Tissue block \\
\hline
\end{tabular}

TABLE 2: MIC (mg/L) of experimental and standard strains.

\begin{tabular}{lccc}
\hline Sample number & MCF & ICZ & AmB \\
\hline ATCC2001 & 0.032 & 0.125 & 0.500 \\
17K1156 & 0.016 & 0.250 & 0.250 \\
J1 & 0.064 & 0.125 & 1.000 \\
17K1173 & 1.000 & 0.500 & 0.500 \\
17K1152 & 0.256 & 0.250 & 1.000 \\
17K1150 & 0.128 & 0.125 & 0.250 \\
16K1132 & 0.032 & 0.500 & 0.500 \\
16K1128 & 0.500 & 0.250 & 2.000 \\
17S709 & 0.064 & 0.0315 & 0.250 \\
16S482 & 0.032 & 0.250 & 0.500 \\
16S506 & 0.016 & 0.0625 & 0.250 \\
\hline
\end{tabular}

The MIC of three drugs in the same species was significantly different $(F=5.066, P<0.05)$. There was no significant difference in the MIC among different strains for the same drug $(F=1.275, P=0.302)$.

and ICZ $(0.125 \mathrm{mg} / \mathrm{L})$, the MICs of only one strain (16K1128) for AmB and two strains (16K1132 and 17K1173) for ICZ were 4 times higher. We found that the MIC values of the four strains were at least 4-fold higher: $17 \mathrm{~K} 1150$ (4fold), $17 \mathrm{~K} 1152$ (8-fold), $16 \mathrm{~K} 1128$ (15-fold), and $17 \mathrm{~K} 1173$ (31-fold).

3.3. Effects of Antifungal Agents on SOD Activity in C. glabrata after Phagocytosis. C. glabrata tends to "hide" or "escape" from killing by phagocytes via the upregulation of the antioxidant system. SOD is at the front line of this antioxidant mechanism, and it has been adopted by many pathogenic bacteria and fungi, including $C$. albicans, Histoplasma capsulatum (H. capsulatum), and Cryptococcus neoformans (C. neoformans). In this study, we found that the SOD activity in C. glabrata phagocytosed by macrophages gradually increased after 1 hour and peaked at 6 hours after infection. As shown in Figure 1, compared with the SOD level of nonphagocytized C. glabrata $(0 \mathrm{~h})$, the SOD level significantly increased at 1 hour after infection $(P<0.05)$. SOD levels in $C$. glabrata cells in macrophages, with or without antifungal treatment, began to decrease at 12 hours after infection. The mean SOD level of fungus-infected macrophages treated with MCF was lower than that of untreated fungus-infected macrophages, especially at 6 and 12 hours after infection $(P<0.05)$. However, there was no significant difference in SOD levels between untreated and fungus-infected macrophages pretreated with ICZ or AmB $(P<0.05)$.

3.4. Cytokines Secreted by C. glabrata-Infected Macrophages. The production of IL-6, IL-8, and TNF- $\alpha$ can be induced by C. glabrata infection $[18,19]$. However, the levels of cytokines secreted by macrophages after C. glabrata infection were significantly lower than those after $C$. albicans infection [20]. In this study, we found that IL-6 increased 5.5-8.4 times within 2-28 hours macrophage infection by C. glabrata, as shown in Figure 2(a) $(F=6562.399$, $P<0.001)$. During the same time period, IL-8 increased 11-27 times (Figure 2(b)) $(F=828.076, P<0.001)$ and TNF$\alpha$ increased $7-10$ times in infected macrophages (Figure 2(c)) $(F=2237.196, P<0.001)$. Interestingly, IL-8 secretion by infected macrophages significantly increased at 24 hours after infection and further increased at 28 hours after infection, whereas IL- 6 and TNF- $\alpha$ gradually increased at 2-28 hours after infection.

Similar to SOD activity, at any point in time, there was no significant difference in IL- 6 and IL- 8 secretion between each antifungal-treated macrophages and untreated macrophages or among the drug pretreatment groups after fungal infection $(P>0.05)$. However, MCF-treated macrophages showed a slight decrease in TNF- $\alpha$ levels within 4-28 hours after infection but were still higher than those in untreated noninfected macrophages $(t=20.611, P<0.05)$.

\section{Discussion}

The innate immune system plays an important role in defending against pathogens, such as the opportunistic pathogen C. glabrata. Infections caused by C. glabrata have become common and cause high morbidity and high mortality. However, there have been fewer studies on its pathogenesis compared with that of C. albicans [21, 22]. Resisting macrophage killing is the first step employed by C. albicans to evade the immune system and cause disseminated infection. Cytokines secreted by macrophages recruit other types of immune cells to the infection site [11]. For example, the mycelium of C. albicans binds to tool-like receptor 2 (TLR2) on monocytes, thereby increasing the level of the anti-inflammatory cytokine IL-10 during infection [23]. Unlike C. albicans, blood infections caused by C. glabrata can persist for a long time in mice without 


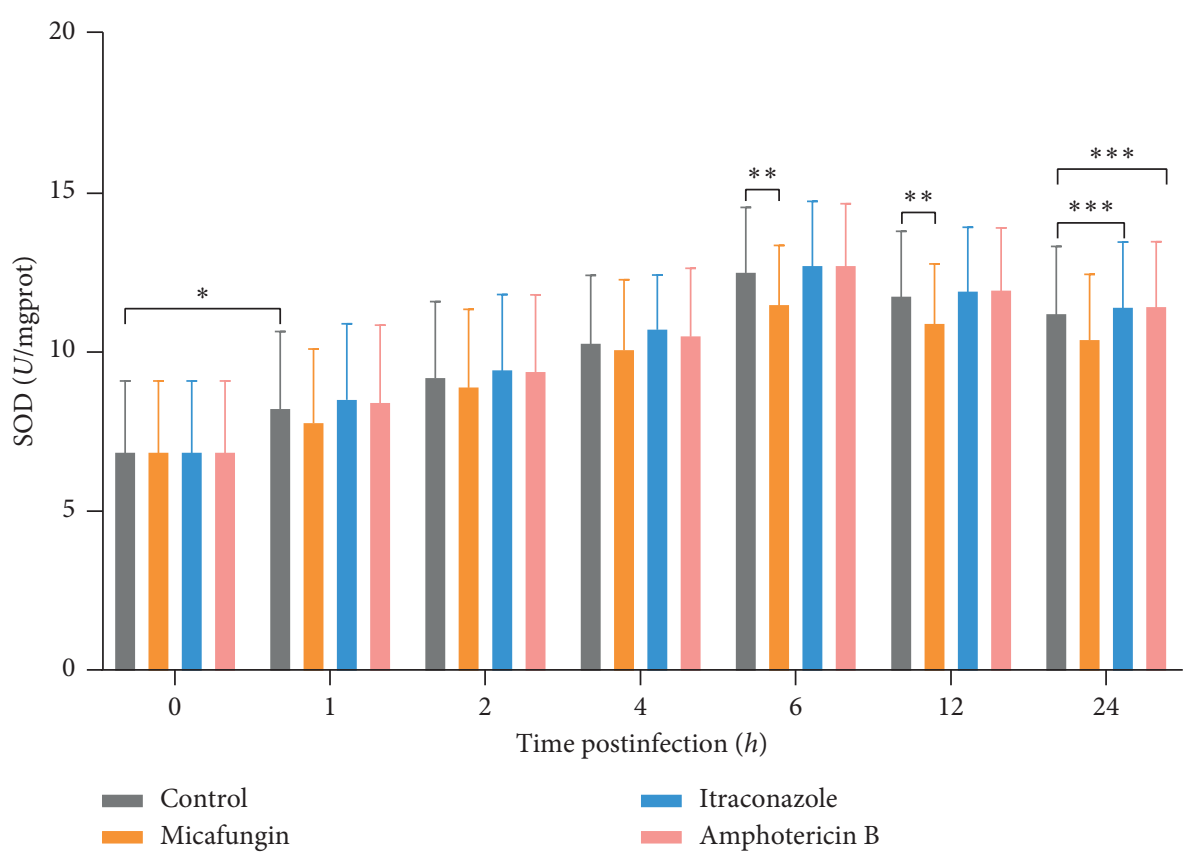

FIGURE 1: SOD activity of C. glabrata in macrophages pretreated with three antifungal drugs. We measured the SOD activity of macrophages treated with MCF, ICZ, and AmB. The control group was not pretreated with antifungal agents. The SOD level after 1 hour was significantly increased compared with the SOD level of nonphagocytized $C$. glabrata ( 0 hour) $\left(^{*}, P<0.05\right)$. The mean SOD level of fungus-infected macrophages treated with MCF was lower than that of untreated fungus-infected macrophages, especially 6-12 hours after infection ( ${ }^{* *}$, $P<0.05)$. There was no significant difference in SOD levels between the untreated and ICZ- or AmB-treated fungus-infected macrophage groups $\left({ }^{* * *}, P>0.05\right)$.

causing high levels of inflammation, even in immunocompetent mice; C. glabrata cannot be eliminated after a few weeks [24]. Our data showed that the levels of IL-6, IL-8, and TNF- $\alpha$ secreted by macrophages significantly increased after infection with C. glabrata, although they were lower than those reported for $C$. albicans infection, which is in consistence to report in previous work [20].

The effects of antimicrobial agents on mammalian cells, especially those of the host innate immune response, have recently been noted, as patients with severe disease may depend on the complex interactions between antibiotics and their activity on pathogens and host cells $[25,26]$. In this study, we selected three antifungal drugs to examine their possible side effects on macrophage function. These three antifungals target different components of fungi. ICZ and AmB target the fungal cell membrane, and MCF inhibits $\beta$-1,3-glucan synthesis for the cell wall [27]. We used a concentration of half the MIC value of each drug to examine its possible effects on cytokine secretion and fungal SOD activity; the results showed that there was no change in the levels of IL- 6 and IL- 8 in antifungal-treated macrophages within 28 hours after fungal infection. In contrast, TNF- $\alpha$ decreased slightly in macrophages treated with MCF. It was reported that cytokines such as IL- 8 and TNF- $\alpha$ are released from human monocytes after exposure to $\beta$-1,3-glucan by C. albicans infection [28]. The reason for the decrease in TNF- $\alpha$ may be that $\beta$-1,3-glucan is insufficient due to the effect of the echinocandin on $\beta$-1,3-glucan synthesis or fungicidal effects. This hypothesis seems to differ from previous observations, which indicated that C. glabrata cells in replicative senescence in macrophages thickened their cell walls when treated with antifungal drugs [29]. The other mechanism may be mediated by hypoxia-induced $\beta$-glucan masking which is dependent on mitochondrial signaling and the cAMP-protein kinase pathway [30]. Since the levels of IL- 8 and IL-6 in macrophages treated with MCF did not change, the possibility of decreasing secondary levels of proinflammatory cytokines secreted by macrophages induced by overexposure to $\beta-1,3$-glucan [31-33] is not yet clear.

Contrary to reports of the antioxidative activity of other fungi induced by ICZ or AmB $[34,35]$, we found that the low concentration of these two antifungal agents had no effect on the SOD activity of phagocytized C. glabrata. Similar to the TNF- $\alpha$ response to MCF-treated macrophages, the SOD activity of phagocytized C. glabrata was reduced when macrophages were pretreated with MCF. Here, the unidentified macrophage effector induced by the echinocandin or the killing effect of the echinocandin is responsible for the decrease in SOD. However, this observation highlights that echinocandins may have antifungal effects by simultaneously inhibiting cell wall formation and reducing antioxidants of C. glabrata.

These results support our hypothesis that echinocandins may directly affect the activity of human cells related to innate immunity. In fact, the effect of echinocandins on cytokines secreted by macrophages is related to the function of the endoplasmic reticulum [36]. However, we still lack adequate evidence to conclude that $\beta$-1,3-glucan from C. glabrata is associated with TNF- $\alpha$ production by 


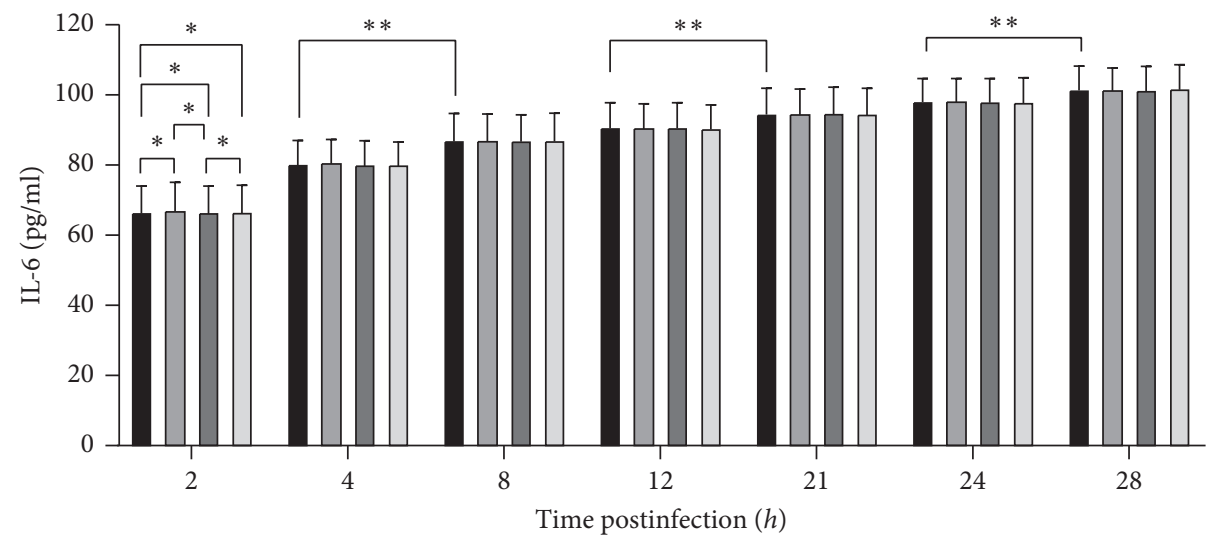

$$
\begin{array}{ll}
\text { Macrophages }+ \text { C. glabrata } & \square \text { MCF-treated macrophages + C. glabrata } \\
\square \text { ICZ-treated macrophages + C. glabrata } & \square \text { AmB-treated macrophages + C. glabrata }
\end{array}
$$

(a)

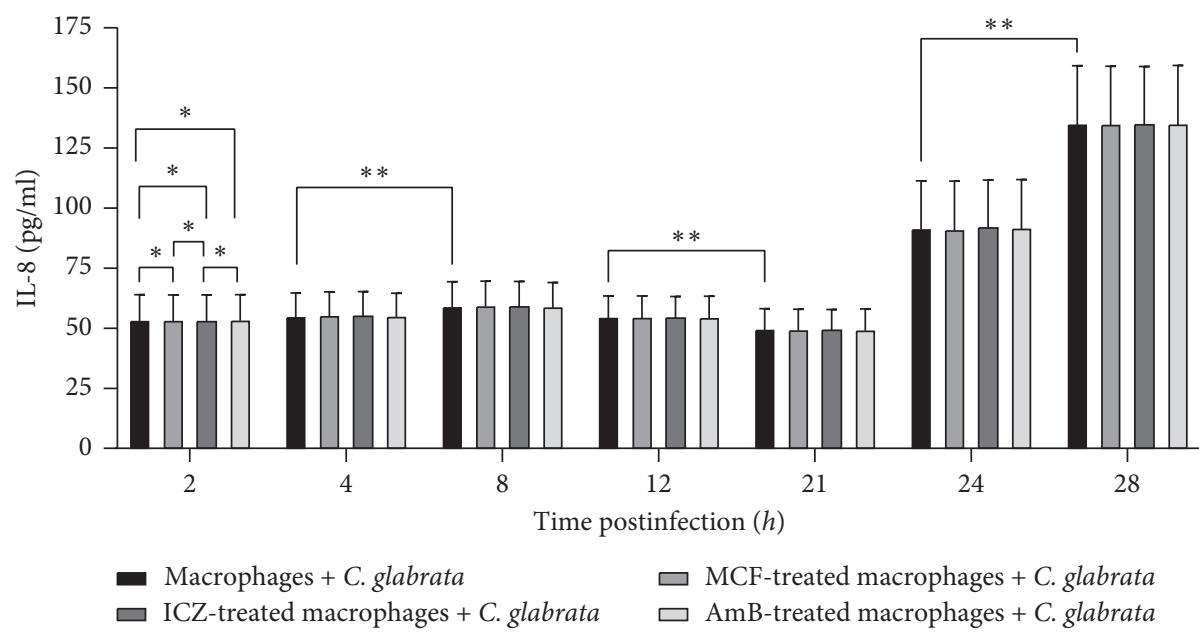

(b)

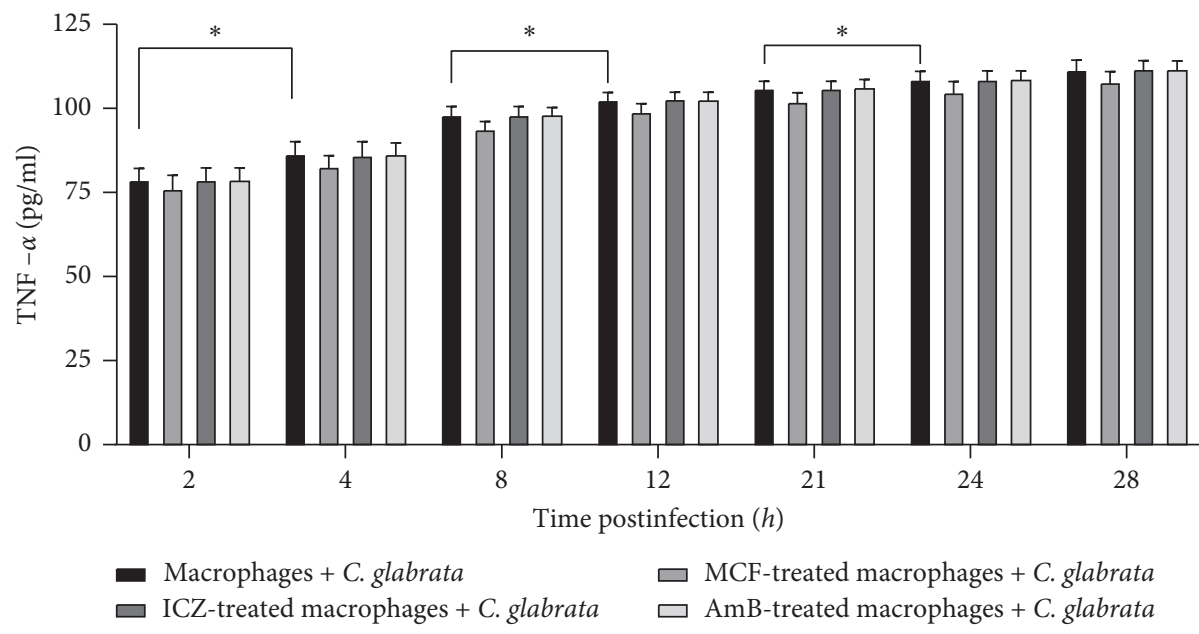

(c)

Figure 2: Effects of different antifungal drugs on the secretion of IL-6, IL-8, and TNF- $\alpha$ from macrophages infected by C. glabrata. The levels of secreted IL-6, IL-8, and TNF- $\alpha$ were determined by ELISA. IL-6 levels secreted by the four groups of macrophages were not statistically significant $\left({ }^{*}, P>0.05\right)$, and IL-6 levels secreted at different time points were significantly different $\left(F=6562.399,{ }^{* *}, P<0.001\right)$. At all-time points, IL-6 levels gradually increased over time (a). IL-8 levels secreted by the four groups of macrophages were not statistically significant $\left({ }^{*}, P>0.05\right)$, and IL-8 levels secreted at different time points were statistically significant $\left(F=828.076,{ }^{* *}, P<0.001\right)$. At all-time points, IL-8 levels fluctuated with time (b). TNF- $\alpha$ levels secreted at different time points were statistically significant $\left(F=2237.196,{ }^{*}, P<0.001\right)$. At alltime points, TNF- $\alpha$ levels gradually increased over time (c). 
macrophages. In addition, this study had several limitations. First, this study used only one dose of antifungal therapy. We evaluated the cytokines secreted by C. glabrata-infected macrophages, and the goal was to compare the difference between treatment with different antifungal drugs and untreated macrophages after treatment with only one concentration (1/2 MIC) of each drug for 24 hours. Small changes in cytokines between cells administered antifungal treatment and no treatment may have resulted from insufficient doses of each drug treatment. Second, the number of C. glabrata cells was not evaluated during the study. We observed that although the total content of antioxidant SOD increased at 6 hours after antifungal treatment, MCF caused a slight decrease in the SOD activity in phagocytized C. glabrata cells. Compared with the other two antifungal drugs and no treatment, the fungicidal effect of MCF may lead to a decrease in C. glabrata cells in macrophages. Third, the amount of fungal sample used in our study was very small. Further studies are required to verify the current results with a larger sample size.

The persistent presence of C. glabrata and the weak inflammatory response in tissues remain one of the biggest challenges in the treatment of systemic fungal infections. As we observed in this study, all clinical specimens were isolated from pus or peritoneal drainage, and none of them were drawn from the blood, again indicating the chronic progression of C. glabrata. In the course of fungal infection, small differences in cytokines from macrophages treated with different antifungal drugs and untreated macrophages confirmed the safety of the three antifungal drugs on the immune response against C. glabrata. Our results also indicate that echinocandins tend to kill fungi via cell wall exposure and interfering with the response of host macrophages.

\section{Summary}

The survival and proliferation of C. glabrata in macrophages is an important factor in systemic infection, which depends on the ability of these fungi to respond effectively to the stressful environment and avoid immune defenses. It has long been recognized that C. glabrata's intrinsic resistance $[37,38]$ to oxidative stress occurs through the high expression of detoxifying enzymes (catalase and superoxide dismutase) during infection [39]. Additionally, resistance to antifungals, including high-value antifungal agents such as echinocandins, has emerged in C. glabrata [40, 41]. Current research is still focused on the interactions between organisms and antibacterial agents, and little is known about the immune effects of antifungals during infection. To understand the effect of antifungal drugs on C. glabratainfected macrophages, we pretreated macrophage cell lines with an azole, echinocandin, and AmB for 24 hours before C. glabrata infection. We found out that macrophages pretreatment with ICZ and AmB had no effect on cytokine production by macrophages or antioxidant SOD activity in phagocytized C. glabrata. However, cytokines and the activity of SOD decreased slightly after macrophages were pretreated with MCF. This study suggests that there is a complex response to antifungal drugs in fungal cells and host defense mechanisms.

\section{Data Availability}

The data used to support the findings of this study are included within the article.

\section{Ethical Approval}

The authors confirm that the ethical policies of the journal, as noted on the journal's author guidelines page, have been adhered to. This study was approved by the review board of First Affiliated Hospital of Kunming Medical University. All experiments were performed following the approved guidelines and regulations and were in accordance with the principles of the Declaration of Helsinki. The experimental protocols were approved by the institutional review board of Kunming Medical University. Patient samples were collected according to protocols approved by the respective institutional review boards.

\section{Consent}

Study participants gave written informed consent in accordance with the Declaration of Helsinki.

\section{Conflicts of Interest}

The authors declare that they have no conflicts of interest.

\section{Authors' Contributions}

Hong-Bin Li, Na Li, and Shu-Ran Wen contributed equally to this work.

\section{Acknowledgments}

This work was supported by the Project for Innovation Team of Yunnan Provincial Science and Technology Department, China (2018HC005), National Natural Science Foundation of China (81560325 and 81660248), and a grant from the Yunnan Provincial Department of Education Science Research Fund Project, Yunnan, China (2018JS211).

\section{References}

[1] M. Nucci, F. Queiroz-Telles, A. M. Tobón, A. Restrepo, and A. L. Colombo, "Epidemiology of opportunistic fungal infections in Latin America," Clinical Infectious Diseases, vol. 51, no. 5, pp. 561-570, 2010.

[2] T. Z. Sampaio Camargo, A. R. Marra, C. V. Silva et al., "Secular trends of candidemia in a tertiary care hospital," American Journal of Infection Control, vol. 38, no. 7, pp. 546-551, 2010.

[3] A. C. Pasqualotto, R. A. Zimerman, S. H. Alves et al., "Take control over your fluconazole prescriptions: the growing importance of Candida glabrata as an agent of candidemia in Brazil," Infection Control \& Hospital Epidemiology, vol. 29, no. 9, pp. 898-899, 2008. 
[4] V. Krcmery and A. J. Barnes, "Non-albicans Candida spp. causing fungaemia: pathogenicity and antifungal resistance," Journal of Hospital Infection, vol. 50, no. 4, pp. 243-260, 2002.

[5] M. A. Pfaller, D. J. Diekema, D. L. Gibbs et al., "Results from the ARTEMIS DISK Global Antifungal Surveillance Study, 1997 to 2007: a 10.5-year analysis of susceptibilities of Candida Species to fluconazole and voriconazole as determined by CLSI standardized disk diffusion," Journal of Clinical Microbiology, vol. 48, no. 4, pp. 1366-1377, 2010.

[6] W. Liu, J. Tan, J. Sun et al., "Invasive candidiasis in intensive care units in China: in vitro antifungal susceptibility in the China-SCAN study," Journal of Antimicrobial Chemotherapy, vol. 69, no. 1, pp. 162-167, 2014.

[7] C. F. Rodrigues, A. Correia, M. Vilanova, and M. Henriques, "Inflammatory cell recruitment in Candida glabrata biofilm cell-infected mice receiving antifungal chemotherapy," Journal of Clinical Medicine, vol. 8, no. 2, 2019.

[8] A. Marcil, D. Harcus, D. Y. Thomas, and M. Whiteway, "Candida albicans killing by RAW 264.7 mouse macrophage cells: effects of Candida genotype, infection ratios, and gamma interferon treatment," Infection and Immunity, vol. 70, no. 11, pp. 6319-6329, 2002.

[9] S. Ghosh, D. H. M. L. P. Navarathna, D. D. Roberts et al., "Arginine-induced germ tube formation in Candida albicans is essential for escape from murine macrophage line RAW 264.7," Infection and Immunity, vol. 77, no. 4, pp. 1596-1605, 2009.

[10] M. C. Lorenz, J. A. Bender, and G. R. Fink, “Transcriptional response of Candida albicans upon internalization by macrophages," Eukaryotic Cell, vol. 3, no. 5, pp. 1076-1087, 2004.

[11] K. Seider, S. Brunke, L. Schild et al., "The facultative intracellular pathogen Candida glabrata subverts macrophage cytokine production and phagolysosome maturation," Journal of Immunology (Baltimore, MD: 1950), vol. 187, no. 6, pp. 3072-3086, 2011.

[12] M. Martchenko, A.-M. Alarco, D. Harcus, and M. Whiteway, "Superoxide dismutases inCandida albicans: transcriptional regulation and functional characterization of the hyphalinducedSOD5Gene," Molecular Biology of the Cell, vol. 15, no. 2, pp. 456-467, 2004.

[13] G. M. Cox, T. S. Harrison, H. C. McDade et al., "Superoxide dismutase influences the virulence of Cryptococcus neoformans by affecting growth within macrophages," Infection and Immunity, vol. 71, no. 1, pp. 173-180, 2003.

[14] C. S. Hwang, G. E. Rhie, J. H. Oh, W. K. Huh, H. S. Yim, and S. O. Kang, "Copper- and zinc-containing superoxide dismutase $(\mathrm{Cu} / \mathrm{ZnSOD})$ is required for the protection of Candida albicans against oxidative stresses and the expression of its full virulence," Microbiology (Reading, England), vol. 148, no. Pt 11, pp. 3705-3713, 2002.

[15] M. Briones-Martin-Del-Campo, E. Orta-Zavalza, I. CanasVillamar et al., "The superoxide dismutases of Candida glabrata protect against oxidative damage and are required for lysine biosynthesis, DNA integrity and chronological life survival," Microbiology (Reading, England), vol. 161, no. Pt 2, pp. 300-310, 2015.

[16] N. C. C. L. S. Reference, Method for Broth Dilution Antifungal Susceptibility Testing of Yeasts; Approved Standard, National Committee for Clinical Laboratory Standards, Villanova, PA, USA, 2nd edition, 2002

[17] M. N. Rai, S. Borah, G. Bairwa, S. Balusu, N. Gorityala, and R. Kaur, "Establishment of an in vitro system to study intracellular behavior of Candida glabrata in human THP-1 macrophages," Journal of Visualized Experiments: JoVE, vol. 82, Article ID e50625, 2013.

[18] S. B. Bazan, B. Walch-Rückheim, M. J. Schmitt, and F. Breinig, "Maturation and cytokine pattern of human dendritic cells in response to different yeasts," Medical Microbiology and Immunology, vol. 207, no. 1, pp. 75-81, 2018.

[19] S. Saegusa, M. Totsuka, S. Kaminogawa, and T. Hosoi, "Cytokine responses of intestinal epithelial-like Caco-2 cells to non-pathogenic and opportunistic pathogenic yeasts in the presence of butyric acid," Bioscience, Biotechnology, and Biochemistry, vol. 71, no. 10, pp. 2428-2434, 2007.

[20] M. Schaller, C. A. Sander, H. C. Korting, R. Mailhammer, G. Grassl, and B. Hube, "Infection of human oral epithelia with Candida species induces cytokine expression correlated to the degree of virulence," Journal of Investigative Dermatology, vol. 118, no. 4, pp. 652-657, 2002.

[21] A. Roetzer, N. Gratz, P. Kovarik, and C. Sch Ã¹/4ler, "Autophagy supportsCandida glabratasurvival during phagocytosis," Cellular Microbiology, vol. 12, no. 2, pp. 199-216, 2010.

[22] S. Brunke, K. Seider, R. S. Almeida et al., "Candida glabratatryptophan-based pigment production via the Ehrlich pathway," Molecular Microbiology, vol. 76, no. 1, pp. 25-47, 2010.

[23] C. A. A. van der Graaf, M. G. Netea, I. Verschueren, J. W. M. van der Meer, and B. J. Kullberg, "Differential cytokine production and Toll-like receptor signaling pathways by Candida albicans blastoconidia and hyphae," Infection and Immunity, vol. 73, no. 11, pp. 7458-7464, 2005.

[24] I. D. Jacobsen, S. Brunke, K. Seider et al., "Candida glabrata persistence in mice does not depend on host immunosuppression and is unaffected by fungal amino acid auxotrophy," Infection and Immunity, vol. 78, no. 3, pp. 1066-1077, 2010.

[25] S. Kanoh and B. K. Rubin, "Mechanisms of action and clinical application of macrolides as immunomodulatory medications," Clinical Microbiology Reviews, vol. 23, no. 3, pp. 590-615, 2010.

[26] T. H. Mogensen, "Pathogen recognition and inflammatory signaling in innate immune defenses," Clinical Microbiology Reviews, vol. 22, no. 2, pp. 240-273, 2009.

[27] T. K. Mazu, B. A. Bricker, H. Flores-Rozas, and S. Y. Ablordeppey, "The mechanistic targets of antifungal agents: an overview," Mini Reviews in Medicinal Chemistry, vol. 16, no. 7, pp. 555-578, 2016.

[28] Y. Nakagawa, N. Ohno, and T. Murai, "Suppression byCandida albicans $\beta$-glucan of cytokine release from activated human monocytes and from T cells in the presence of monocytes," The Journal of Infectious Diseases, vol. 187, no. 4, pp. 710-713, 2003.

[29] T. Bouklas, L. Alonso-Crisostomo, T. Szekely Jr. et al., "Generational distribution of a Candida glabrata population: resilient old cells prevail, while younger cells dominate in the vulnerable host," PLoS Pathogens, vol. 13, no. 5, Article ID e1006355, 2017.

[30] A. Pradhan, G. M. Avelar, J. M. Bain et al., "Hypoxia promotes immune evasion by triggering beta-glucan masking on the Candida albicans cell surface via mitochondrial and cAMPprotein kinase A signaling," mBio, vol. 9, no. 6, 2018.

[31] M. G. Netea, N. A. Gow, C. A. Munro et al., "Immune sensing of Candida albicans requires cooperative recognition of mannans and glucans by lectin and Toll-like receptors," Journal of Clinical Investigation, vol. 116, no. 6, pp. 1642-1650, 2006. 
[32] G. D. Brown and S. Gordon, "Fungal $\beta$-glucans and mammalian immunity," Immunity, vol. 19, no. 3, pp. 311-315, 2003.

[33] G. D. Brown and S. Gordon, "Immune recognition of fungal $\beta$-glucans," Cellular Microbiology, vol. 7, no. 4, pp. 471-479, 2005.

[34] C. E. B. Linares, S. R. Giacomelli, D. Altenhofen, S. H. Alves, V. M. Morsch, and M. R. C. Schetinger, "Fluconazole and amphotericin-B resistance are associated with increased catalase and superoxide dismutase activity in Candida albicans and Candida dubliniensis," Revista da Sociedade Brasileira de Medicina Tropical, vol. 46, no. 6, pp. 752-758, 2013.

[35] C. F. Hoehamer, E. D. Cummings, G. M. Hilliard, and P. D. Rogers, "Changes in the proteome of Candida albicans in response to azole, polyene, and echinocandin antifungal agents," Antimicrobial Agents and Chemotherapy, vol. 54, no. 5, pp. 1655-1664, 2010.

[36] U. K. Rapp and S. H. E. Kaufmann, "Glucose-regulated stress proteins and antibacterial immunity," Trends in Microbiology, vol. 11, no. 11, pp. 519-526, 2003.

[37] M. Cuellar-Cruz, M. Briones-Martin-del-Campo, I. CanasVillamar et al., "High resistance to oxidative stress in the fungal pathogen Candida glabrata is mediated by a single catalase, Cta1p, and is controlled by the transcription factors Yap1p, Skn7p, Msn2p, and Msn4p," Eukaryotic Cell, vol. 7, no. 5, pp. 814-825, 2008.

[38] D. Kaloriti, A. Tillmann, E. Cook et al., "Combinatorial stresses kill pathogenicCandidaspecies," Medical Mycology, vol. 50, no. 7, pp. 699-709, 2012.

[39] A. J. Brown, K. Haynes, and J. Quinn, "Nitrosative and oxidative stress responses in fungal pathogenicity," Current Opinion in Microbiology, vol. 12, no. 4, pp. 384-391, 2009.

[40] G. Butler, M. D. Rasmussen, M. F. Lin et al., "Evolution of pathogenicity and sexual reproduction in eight Candida genomes," Nature, vol. 459, no. 7247, pp. 657-662, 2009.

[41] M. I. Hood and E. P. Skaar, "Nutritional immunity: transition metals at the pathogen-host interface," Nature Reviews Microbiology, vol. 10, no. 8, pp. 525-537, 2012. 